\title{
Adolescentes usuarios de sustancias ingresados en tratamiento: características y factores asociados a la duración del tratamiento
}

\section{Substance-using adolescents admitted to inpatient treatment: characteristics and factors associated with length of time in treatment}

\author{
Alexandre Quelho Comandule*,**, Maria de Fatima Rato Padin**,***, \\ Martha Canfield $* * * *$, Ronaldo LaranjeIra****** \\ * Santa Casa de Misericordia de Sorocaba, Sorocaba, São Paulo. Brasil. ** Instituto Nacional de Políticas Públicas de \\ Alcohol y otras Drogas, São Paulo. Brasil. *** Departamento de Psiquiatría. Universidad Federal de São Paulo, São Paulo. \\ Brasil. **** Sección de Psicología de la Salud, Departamento de Psicología, Instituto de Psiquiatría, Psicología y Neurociencia. \\ King's College London, Londres. Reino Unido.
}

\section{Resumen}

Los estudios sobre adolescentes ingresados para tratamiento de uso de sustancias son limitados. Analizamos las características de adolescentes ingresados para tratamiento en un hospital psiquiátrico en Brasil y los factores asociados con la duración de su tratamiento. Métodos: estudio observacional retrospectivo. Analizamos los registros electrónicos de tratamiento de 172 jóvenes (hasta los 17 años de edad) ingresados para tratamiento por uso de sustancias en el Hospital Lacan en Brasil. Resultados: La edad media de los participantes era 15,18 años $(\mathrm{SD}=1,39)$. La muestra era mayoritariamente masculina $(68,60 \%)$, cuyo ingreso fue involuntario $(80,81 \%)$, sin escolarizar $(89,82 \%)$, involucrada en el sistema de justicia penal $(59,88 \%)$ y proveniente de una familia con problemas relacionados con el uso de sustancias $(74,67 \%)$. La readmisión como paciente a tratamiento por problemas de uso de sustancias era frecuente. Como media, los adolescentes estuvieron ingresados para tratamiento durante 3 meses. La duración del tratamiento estaba asociada con: admisión involuntaria al tratamiento, reingreso hospitalario para tratamiento, solicitudes de alta del tratamiento por parte de familiares/cuidadores, nivel de estudios, abandono escolar debido a conducta agresiva, y uso de cocaína. Conclusión: Los hallazgos destacan los perfiles complejos de los adolescentes ingresados para tratamiento por el uso de sustancias en Brasil. Es necesaria una colaboración entre los sistemas de salud mental, educación y servicios de justicia para tratar el uso de sustancias entre adolescentes.

Palabras clave: Adolescentes; Tratamiento hospitalario; Uso de sustancias.

\begin{abstract}
Studies of adolescents receiving inpatient substance use treatment remain limited. We explored the characteristics of adolescents who received substance use treatment as inpatients in a psychiatric hospital in Brazil and factors associated with length of time in this treatment. Methods: A retrospective observational study was performed. Electronic treatment records of 172 young people (aged 17 and below) receiving substance use treatment at Hospital Lacan in Brazil were analysed. Results: The mean age of participants was 15.18 years $(\mathrm{SD}=1.39)$. The sample was characterised as predominately male $(68.60 \%)$, who entered treatment involuntarily $(80.81 \%)$, were out of school $(89.82 \%)$, were involved with the criminal justice system $(59.88 \%)$ and came from a family with substance use problems (74.67\%). Re-admission to inpatient treatment for substance use problems was common. On average, adolescents received inpatient treatment for 3 months. Length of time in treatment was associated with: involuntary admission to treatment, re-admission to inpatient treatment, requests of discharge from treatment by a relative/ caretaker, education level, leaving school due to aggressive behaviours and cocaine use. Conclusion: Findings highlight the complex profiles of adolescents receiving substance use treatment in Brazil. Crosssystem collaboration between mental health, educational and justice services are needed to treat adolescents' substance use. Keywords: Adolescents; Inpatient treatment; Substance use.
\end{abstract}


A

pesar de la evidencia de una disminución del uso de sustancias entre los jóvenes en algunos países (Broadfield, 2017; Dennis y Scott, 2007; Miech, Johnston, O’Malley, Bachman y Schulenberg, 2016; Plettinckx et al., 2014), la admisión a tratamiento de adolescentes con trastornos de uso de alcohol y de otras sustancias continúa aumentando (Roxburgh y Burns, 2013). En Brasil, la estimación de la prevalencia de uso de sustancias entre adolescentes es alta: $42,4 \%$ de alcohol, 9,6\% de tabaco y 25,5\% de sustancias ilegales (Carlini et al., 2010; Malta et al., 2011). Las sustancias ilegales más usadas entre adolescentes brasileños son cannabis e inhalables/disolventes (Carlini et al., 2010; Madruga et al., 2012). Según la encuesta nacional más reciente sobre el uso de cocaína-crack en Brasil, los jóvenes menores de 18 años representan aproximadamente el 14\% de los usuarios habituales de cocaína-crack (Bastos y Bertoni, 2014). En la actualidad, no hay datos disponibles respecto de la prevalencia de adolescentes ingresados para tratamiento del uso de sustancias en Brasil (Vasters y Pillon, 2011). La evidencia hallada en la literatura internacional sugiere que es menos probable que adolescentes, en comparación con adultos, busquen tratamiento (Cornelius et al., 2003; Sussman, Skara y Ames, 2009; Winters, Tanner-Smith, Bresani y Meyers, 2014). Las cifras de los Estados Unidos muestran que solo el $10 \%$ de los adolescentes con problemas por uso de sustancias son admitidos a tratamiento (Substance Abuse and Mental Health Services Administration, 2013).

De manera similar a otros países, se han realizado intentos en Brasil para ofrecer servicios de tratamiento que reconozcan la etapa de desarrollo psicológica y física (Castellanos-Ryan, O'Leary-Barret y Conrod, 2013; Winters et al., 2014) y los problemas familiares específicos que puedan estar asociados con el uso de sustancias por parte de los adolescentes (Bertrand et al., 2013; Coatsworth, Santisteban, McBride y Szapocznik, 2001). No obstante, es común que a los adolescentes se les incluya en programas que son meras modificaciones de programas para adultos. El consenso es claro tanto en la literatura brasileña como en la internacional que los servicios exclusivamente para adolescentes representan un umbral mínimo de la calidad de los tratamientos para el uso de sustancias (Lopes, Nobrega, Del Prette y Scivoletto 2013; NIDA, 2014) y que las diferencias en cuanto a la adherencia al tratamiento y en los resultados del mismo pueden ser, en gran parte, explicados por las características individuales de los clientes (Knudsen, 2009). En la actualidad, hay una gran demanda en Brasil de tratamientos para el uso de sustancias que sean personalizados a las necesidades de los adolescentes consumidores (Vasters y Pillon, 2011). No hay datos sobre las características de aquellos adolescentes brasileños ingresados para tratamiento por uso de sustancias y las posibles asociaciones entre las mismas y los resultados de los servicios.
Nuestro estudio tuvo como fin empezar a abordar estos temas mediante la descripción de las características de los adolescentes ingresados para tratamiento en un hospital psiquiátrico en Brasil. Dado que estudios anteriores han mostrado que la duración de los programas de pacientes ingresados está asociada con los resultados de los tratamientos, incluyendo la recaída cuando vuelven a su entorno natural (Battjes, Gordon, O’Grady, Kinlock, y Carswe1l, 2003; Chung y Maisto, 2006), un segundo objetivo de nuestro estudio fue identificar los factores asociados con la duración del tratamiento hospitalario. La evidencia sugiere que la duración del tratamiento varía según la severidad del problema de uso de sustancias del adolescente (Battjes, Gordon, O’Grady, y Kinlock, 2004; Winters et al., 2018). Se ha asociado la dependencia severa al uso de sustancias entre los jóvenes con una variedad de fuerzas complejas, incluyendo el funcionamiento familiar, malas compañías y problemas escolares (Serrano et al., 2018; Svensson, 2000; Sussman et al., 2009). Los estudios sobre los factores asociados con la duración del tratamiento hospitalario entre adolescentes son limitados. Esperamos que este estudio sirva como punto de partida para implementar programas integrales, específicos y personalizados para adolescentes con problemas por uso de sustancias en Brasil, y que, a la vez, sea una luz en la literatura internacional respecto de los factores asociados con la duración de los tratamientos hospitalarios para uso de sustancias entre adolescentes.

\section{Métodos}

Realizamos un estudio observacional retrospectivo. Los datos fueron recopilados de los registros de 172 jóvenes (hasta los 17 años de edad) derivados al Hospital Psiquiátrico Lacan en la ciudad de São Paulo, entre los años 2014 y 2016. Estas instalaciones de tratamiento gestionadas por un acuerdo entre el Sistema de Salud Pública brasileña (SUS) y financiación filantrópica, ofrece servicios hospitalarios sin coste (i.e., atención psiquiátrica, psicológica y ocupacional) para poblaciones de jóvenes y adultos con trastornos psiquiátricos, incluyendo dependencia al uso de sustancias. Analizamos los datos de todos los pacientes jóvenes referidos a Lacan debido a problemas de uso de sustancias, con/sin otros problemas psiquiátricos. Los pacientes podían haber sido admitidos de manera voluntaria o involuntaria (derivaciones y presiones de familiares o del sistema de justicia penal). El servicio para usuarios de sustancias en Lacan está diseñado para promover la abstinencia de las sustancias, clarificar los diagnósticos, y determinar u optimizar la medicación. Como parte de la intervención médica, los pacientes ingresados en Lacan participan en terapia individual y familiar. Los pacientes no pueden dejar el servicio; ni siquiera para asistir a la escuela. Tras su hospitalización, los pacientes son derivados a la red de salud mental disponible en sus ciudades de 
residencia. El comité ético de la Universidad Federal de São Paulo aprobó el estudio. Para proteger la privacidad y confidencialidad de los pacientes, los gestores de datos del hospital transfirieron datos anónimos al equipo de investigación.

\section{Análisis}

Todos los datos fueron recopilados como parte de la valoración rutinaria y el tratamiento de los adolescentes en el Hospital Lacan.

Características sociodemográficas: En el contacto inicial con el servicio, se recopilaba información sobre edad, sexo, y nivel de estudios de los participantes en el momento de su ingreso. Cuando el adolescente no estaba escolarizado en el momento de su ingreso, se le preguntaba el motivo. También se recopilaban datos respecto de las personas con las cuales convivían los pacientes (i.e., madres solo, abuelos, hogar no familiar).

Características del tratamiento: Los variables del tratamiento incluyeron el número de días en tratamiento, y si el alta del tratamiento actual fue concedida por el servicio o bajo petición de un familiar/cuidador, datos habitualmente registrados por parte del servicio. En la primera valoración clínica, también se recogieron datos sobre cómo el paciente ingresó al tratamiento (i.e., voluntariamente o involuntariamente - debido a referencias y presiones de escuelas, familiares o del sistema de justicia penal), el número de admisiones a tratamientos hospitalarios, y el tiempo transcurrido desde el tratamiento anterior.

Características clínicas: El diagnóstico principal de la CIE10 se registra habitualmente en un campo estructurado. Los datos fueron extraídos para todos aquellos pacientes con problemas de uso de sustancias como diagnóstico principal o secundario. Datos sobre patrones de uso de sustancias fueron extraídos de la valoración clínica inicial - o, en algunos casos, durante la primera o segunda consulta con el psicólogo clínico - usando un instrumento estructurado, diseñado por el servicio. El mismo preguntaba sobre los tipos de sustancias usadas y la frecuencia de uso (i.e., esporádico, casi diario, diario, una vez por día, dos veces por día). Los participantes fueron codificados como usuarios de una sustancia específica si reportaron uso diario de la misma, independientemente de frecuencia. A los participantes también se les pidió su edad cuando iniciaron el uso de sustancias legales e ilegales, y si conocían la existencia de problemas con el uso de sustancias entre otros miembros de su familia. Los participantes fueron codificados con historial de trastorno de salud mental previo a su ingreso para tratamiento si se registró en la valoración clínica inicial. Se registraron informes de trastorno de salud mental en el momento del ingreso al tratamiento para los participantes que indicaron un trastorno de salud mental no necesariamente asociado con uso de sustancias en la CIE-10.

Características penales: Los variables de justicia penal incluyeron historial de involucración en el sistema de justicia penal, tráfico de drogas y robo para mantener el uso de sustancias. Toda esta información se valoró durante el contacto inicial con el servicio, usando un instrumento estructurado, diseñado por el servicio.

\section{Análisis de datos}

Calculamos estadísticas descriptivas usando frecuencias y porcentajes para los datos categóricos, las medias y las desviaciones estándar para variables continuas. Creamos una variable ordinal para duración temporal del tratamiento de 1 a 5 , según los siguientes días en tratamiento: hasta 30 días; 31-60 días; 61-90 días; 91-120 días; 121-255 días. Calculamos la mediana y el rango intercuartílico. Los factores asociados con la duración temporal del tratamiento se consideraron usando modelos de regresión logística ordinal. Informamos la razón de momios (OR) y el intervalo de confianza (IC) de $95 \%$ de modelos no ajustados y ajustados (controlando por la edad y el sexo de los participantes).

\section{Resultados}

La edad media de los participantes era 15,18 años (SD $=1,39)$. La mayoría de los participantes eran hombres $(68,60 \%)$, ingresaron al tratamiento de manera involuntaria $(80,81 \%)$ y no asistían a la escuela en el momento de su ingreso $(89,82 \%)$. De los mismos sin escolarizar, el $62,18 \%$ informó uso de sustancias como el motivo de su abandono escolar. Casi todos los pacientes fueron referidos a atención continua tras su tratamiento hospitalario: el $54,01 \%$ a servicios contra la adicción; el 25\% a servicios para niños y adolescentes; y el 19,02\% a servicios de salud mental. Los historiales de involucración en el sistema de justicia penal fueron muchos $(59,88 \%)$. El reingreso como paciente a tratamiento hospitalario era frecuente $(38,36 \%)$, con una media de 3 años para readmisión. Las siguientes características se reportaron con frecuencia: trastorno de salud mental en el momento de la admisión (25\%; los trastornos mentales más comunes informados incluyeron trastorno por déficit de atención con hiperactividad y trastornos del estado de ánimo), involucración en tráfico de drogas $(37,87 \%)$, robo $(22,10 \%)$ y convivencia con la madre $(41,52 \%)$. Tres cuartas partes de la muestra informó tener un miembro familiar con problemas de uso de sustancias $(74,67 \%)$ y que dicho familiar era miembro directo de la familia $(75,89 \%)$.

Casi todos los participantes informaron del uso de cannabis $(97,09 \%)$ y el uso diario del mismo $(89,51 \%)$. También era elevado el uso de alcohol y tabaco $(88,37 \%$ 
Tabla 1. Variables monofactoriales asociadas con la duración temporal del tratamiento hospitalario $(N=172)$

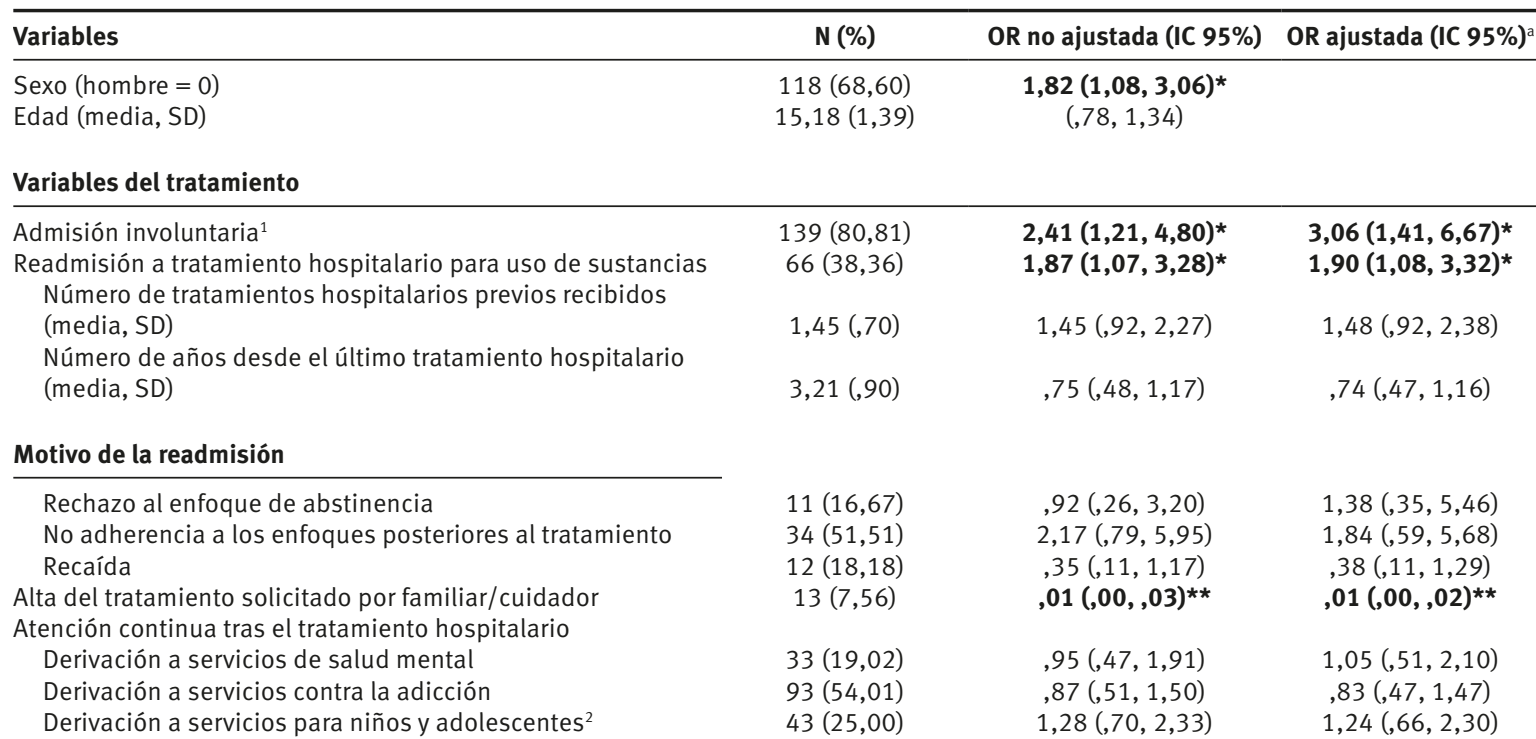

\section{Variables psicológicas}

Historial de trastorno mental previo a la admisión a tratamiento

Reporte de trastorno mental en el momento

de la admisión a tratamiento ${ }^{3}$

$19(11,5)$

, $51(, 19,1,31)$

,49 $(, 19,1,61)$

$43(25,00)$

$1,12(, 59,2,12)$

$1,26(, 66,2,41)$

\section{Variables relacionadas con los estudios}

\begin{tabular}{|c|c|c|c|}
\hline $\begin{array}{l}\text { Nivel de estudios superiores (escuela primaria }=0 \text { ) } \\
\text { Abandono escolar } \\
\text { Abandono escolar debido al uso de sustancias } \\
\text { Abandono escolar debido a conducta agresiva }\end{array}$ & $\begin{array}{l}97(58,79) \\
150(89,82) \\
74(49,33) \\
11(7,33)\end{array}$ & $\begin{array}{c}, 68(, 49,, 95)^{*} \\
, 80(, 34,1,90) \\
, 88(, 44,1,72) \\
3,42(1,10,10,61)^{*}\end{array}$ & $\begin{array}{c}, 62(, 44,, 89)^{*} \\
, 81(, 34,1,94) \\
, 85(, 43,1,68) \\
\mathbf{3 , 4 2 ( 1 , 0 1 , 9 , 8 7 ) *}\end{array}$ \\
\hline Variables relacionadas con el sistema penal & & & \\
\hline $\begin{array}{l}\text { Historial de involucración en el sistema de justicia penal } \\
\text { Historial de asistencia a unidades de atención a la } \\
\text { delincuencia juvenil } \\
\text { Involucración en el tráfico de drogas para mantener el uso de } \\
\text { sustancias } \\
\text { Involucración en robo para mantener el uso de sustancias }\end{array}$ & $\begin{array}{l}103(59,88) \\
39(37,86)\end{array}$ & $\begin{array}{l}1,24(, 71,2,16) \\
, 78(, 38,1,60)\end{array}$ & $\begin{array}{l}1,47(, 83,2,61) \\
, 71(, 34,1,51)\end{array}$ \\
\hline
\end{tabular}

\section{Familia}

Convivencia con

Padre con/sin hermanos $13(7,60)$

Abuelos con/sin hermanos $16(9,36)$

Ambos padres $36(21,05)$

Hogar no familiar ${ }^{5}$

Problema de uso de sustancias entre miembros de la familia $\quad 112(74,67)$

Familiar directo usuario de sustancias

(madre/padre/hermanos)

$85(75,89)$

,56 (,32, ,97)*

$1,72(, 67,4,42)$

$1,32(, 54,3,19)$

, $80(, 39,1,62)$

$1,91(1,01,3,65)^{*}$

$2,38(1,20,4,76)$ *

$2,71(1,08,6,80)$ *
,62 (,35, 1,12)

$1,58(, 62,4,50)$

$1,21(, 49,2,99)$

, $83(, 40,1,70)$

$1,59(, 81,3,15)$

$2,15(1,06,4,37)$ *

$2,61(1,02,6,65)^{*}$

\section{Variables de uso de sustancias}

\begin{tabular}{|c|c|c|c|}
\hline Uso de alcohol & $152(88,37)$ &, $62(, 23,1,84)$ &, $82(, 24,2,79)$ \\
\hline Uso diario de alcohol & $44(28,94)$ & $1,31(, 68,2,51)$ & $1,22(, 63,2,35)$ \\
\hline Uso de tabaco & $135(78,49)$ & $1,35(, 69,2,62)$ & $1,29(, 66,2,50)$ \\
\hline Uso diario de tabaco & $124(91,85)$ & ,99 (,99, 1,01) & ,99 $(, 99,1,00)$ \\
\hline Uso diario de cannabis & $128(76,64)$ & $1,65(, 62,4,39)$ & $1,60(, 61,4,26)$ \\
\hline Uso de cocaína & $139(80,81)$ & $2,31(1,13,4,72)^{*}$ & $2,19(1,06,4,53)$ * \\
\hline Uso diario de cocaína & $100(71,94)$ &, $87(, 42,1,80)$ & $1,26(, 49,2,16)$ \\
\hline Inicio de uso de sustancias (media, SD) & $12,03(1,72)$ &, $80(, 59,1,07)$ &, $81(, 60,1,10)$ \\
\hline
\end{tabular}

Nota. Los números en negrita significan valores $p$ de ${ }^{*} p<, 05,{ }^{* *} p<, 001 .{ }^{a}$ Modelo ajustado para sexo y edad. ${ }^{1}$ Involuntaria incluye familia y orden judicial. ${ }^{2}$ Servicios para niños y adolescentes incluyendo servicios de salud mental con esta población como diana. ${ }^{3}$ Trastornos mentales no necesariamente asociados con el uso de sustancias. ${ }^{4}$ Abandono/expulsión/exclusión permanente/desistimiento/ de la escuela. ${ }^{5}$ Residencia en orfanato/establecimiento correccional de menores/sin techo. 
y $78,49 \%$, respectivamente). Más de una cuarta parte de los que bebían alcohol lo hacían a diario $(28,94 \%)$. Una gran proporción de la muestra informó del uso de cocaína $(80,81 \%)$ y el uso diario de la misma $(71,94 \%)$. Más de una cuarta parte de la muestra informó del uso de cocaína-crack $(29,07 \%)$ y, de aquellos que informaron el uso de esta sustancia, el $72 \%$ la usaba a diario. La edad media de inicio en el uso de sustancias fue de 12,03 (SD = 1,72) años de edad.

\section{Variables asociadas con la duración temporal del tratamiento hospitalario}

El número de días en tratamiento varió entre 2 y 255, con una media de entre 61-90 días ( $\mathrm{RIC}=31-60$ días $)$. El análisis monofactorial (Tabla 1) mostró que la probabilidad de menor tiempo en tratamiento estaba asociada con peticiones de alta del tratamiento por parte de un familiar/cuidador, nivel de estudios más alto y convivencia con la madre. No obstante, un mayor tiempo en tratamiento estaba asociado con ser mujer, ingreso involuntario al tratamiento, readmisión como paciente hospitalizado, abandono escolar debido a conducta agresiva, vivir en un hogar no familiar, y el uso de cocaína.

Después de controlar por edad y sexo de los participantes, las variables siguientes aún se asociaban con la duración temporal del tratamiento: admisión involuntaria al tratamiento, reingreso como paciente hospitalizado, solicitudes de alta del tratamiento por parte de familiares/ cuidadores, nivel de estudios, abandono escolar debido a conducta agresiva, y el uso de cocaína.

\section{Discusión}

Este estudio muestra que los problemas de uso de sustancias en una cohorte de adolescentes brasileños ingresados para tratamiento en un hospital psiquiátrico están asociados con un amplio rango de problemas. La mayoría de los pacientes había abandonado la escuela, estado involucrados con el sistema de justicia penal, sido admitida a tratamiento de manera involuntaria, y venía de familias con miembros que usaban sustancias. La readmisión como paciente a tratamiento era frecuente, igual que la involucración en tráfico de drogas. Aunque la mayoría de los adolescentes convivía con uno o ambos padres biológicos, casi una cuarta parte de la muestra vivía fuera del hogar familiar (i.e., en la calle y en centros penales de rehabilitación). Casi todos los participantes informaron del uso de alcohol y tabaco. El cannabis fue la sustancia ilegal más frecuentemente usada. Casi todos los participantes también informaron del uso de cocaína. El uso de cocaína-crack era frecuente y, entre aquellos que informaron el uso de esta sustancia, casi las tres cuartas partes la usaba a diario. La edad media de inicio en el uso de sustancias fue de 12 años de edad. Nuestros resultados amplían los hallazgos de otros estudios realizados en otros lugares que sugieren que los adolescentes ingresados por tratamiento hospitalario tienen problemas severos de uso de sustancias, altas tasas de recaída, y problemas familiares, escolares y legales (Battjes et al., 2003; Chung y Maisto, 2006).

Como media, los adolescentes de nuestro estudio estuvieron en tratamiento hospitalario durante 3 meses (61-90 días), una cifra significativamente más alta que la recomendación internacional de hasta 30 días para adolescentes (Paino, Aletraris y Roman, 2015). Los motivos por esto no están claros, pero el hecho que el Hospital Lacan está parcialmente financiado por fondos filantrópicos permite mayor flexibilidad respecto de la duración temporal, en comparación con los servicios del Sistema de Salud Pública brasileña. Además, el $80 \%$ de nuestra muestra ingresó a tratamiento de manera voluntaria (por derivaciones y presiones de familiares o del sistema de justicia penal). La falta de motivación o de preparación para recibir tratamiento también podía haber supuesto barreras adicionales ante el inicio de cambios en la conducta de los adolescentes y su compromiso con el tratamiento (Battjes et al., 2003; Battjes et al., 2004; Mensinger, Diamond, Kaminer y Wintersteen, 2006; Paino et al., 2015). En nuestro estudio, un mayor tiempo en tratamiento estaba asociado con ser mujer, ingreso involuntario, readmisión como paciente para tratamiento hospitalario, menor nivel de estudios, no convivencia con la madre biológica, vivir en un hogar no familiar, tener un miembro de la familia que usa sustancias, y el uso de cocaína. Estudios anteriores han mostrado que era más probable que los adolescentes que estuvieron en tratamiento hospitalario más tiempo tuvieran resultados más favorables (Chung y Maisto, 2006; Knudsen, 2009). No obstante, no está claro si la direccionalidad de esta asociación es igual entre adolescentes ingresados para tratamiento en un hospital psiquiátrico. Es importante señalar que el tratamiento para uso de sustancias ofrecido en el Hospital Lacan se centra únicamente en problemas de uso de sustancias. Este servicio carece de enfoques diseñados para integrar otros elementos del contexto social de los adolescentes, incluyendo, por ejemplo, apoyo educativo. La literatura ha establecido, de manera sólida, que el aislamiento y el fracaso escolar podrían aumentar el rechazo social en la escuela, que, a su vez, podría aumentar los vínculos con malas compañías fuera de la escuela, el uso de sustancias, un bajo rendimiento académico, y el desarrollo de problemas legales más tarde en la vida (Bachman et al., 2008; Henry, Knight y Thornberry, 2012; Li y Lerner, 2011). Además, se han identificado compañeros que usan drogas y problemas académicos como factores de riesgo de recaída para jóvenes tras un tratamiento para el uso de drogas (Clark y Winters, 2002; Svensson, 2000). Nuestros hallazgos llaman la atención a la importancia de considerar variaciones respecto de las necesidades educativas, problemas psicológicos y el funcionamiento familiar 
de los adolescentes. Una dirección importante de estudios futuros podría ser investigar la relevancia de estos factores de riesgo para los resultados tras tratamiento para uso de sustancias en adolescentes. La información sobre estos factores de riesgo son prometedores para la implementación de valoraciones integrales que podrían impactar la programación y el tipo de intervenciones a implementar.

Otro hallazgo que requiere de mayor estudio es la media de 3 años para aquellos participantes en tratamientos hospitalarios con anterioridad antes de su readmisión a tratamiento para el uso de sustancias. Los estudios sobre la recaída entre adolescentes que habían sido admitidos a programas hospitalarios sugieren que uno de tres adolescentes que tienen recaída a cualquier patrón de uso de sustancias tras el tratamiento retornan a un uso intenso o al nivel de uso de sustancias previo al tratamiento tras 1-2 años de seguimiento (Myers, Brown, y Mott, 1995; Winters, Stinchfield, Opland, Weller y Latimer, 2000). Mientras que nuestra variable únicamente permite especular acerca de las características de la recaída, señala la necesidad de valorar los puntos de recaída de los adolescentes en recuperación tras su tratamiento, y los posibles mecanismos que pueden subyacer a dichos puntos de cambio. Son necesarios estudios empíricos para determinar cómo eventos específicos en la vida, influencias contextuales y temas inherentes a las etapas vitales pueden afectar la recaída al uso de sustancias entre adolescentes en Brasil. La integración de la recaída en los marcos de tratamiento debería fundamentarse sobre los procesos causantes de la recaída (Chung y Maisto, 2006) y deberían implementarse procedimientos para un seguimiento coherente de los adolescentes tras su ingreso.

Nuestros hallazgos también destacan la necesidad de unas directrices robustas nacionales para un modelo de atención continua para adolescentes en Brasil. Nuestros datos sugieren una inconsistencia sobre derivaciones a atención continua tras tratamiento hospitalario para uso de sustancias, dado que algunos adolescentes son derivados a servicios contra la adicción, otros a servicios de salud mental y algunos a servicios para niños y adolescentes. La evidencia que la mayoría de los participantes readmitidos a tratamiento hospitalario debido a problemas tras su tratamiento ambulatorio demuestra la urgencia de contar con enfoques sobre aspectos conductuales específicos que tengan como fin vincular, retener y adherir a los adolescentes a la atención continua. Esto podría incluir, por ejemplo, definir el tipo de servicios a los cuales los adolescentes con problemas de uso de sustancias han de ser derivados, desarrollar un enfoque de tratamiento multifacético impartido por personal cualificado que aborde todos los aspectos de la vida de los adolescentes (e.g., escuela, hogar, y actividades públicas) (Kelly, Myers y Brown, 2000; Winters et al., 2014) y ofrecer atención continua desde enfoques divulgativos para facilitar la involucración de las familias de los adolescentes en los programas (e.g., visitas domiciliarias y llamadas telefónicas).

Algunas limitaciones de nuestro estudio incluyen el tamaño muestral relativamente pequeño, que puede haber afectado la representatividad de nuestros hallazgos y la asociación entre los variables del análisis de regresión. No fue posible realizar comparaciones entre duración temporal del tratamiento y cada tipo de sustancia usada debido al solapamiento considerable entre grupos según el tipo de sustancia consumida. Aunque registramos los informes de trastorno mental, no fue posible obtener detalles fiables sobre el diagnóstico debido al sesgo de información científica en el uso de registros electrónicos. Por ejemplo, la valoración clínica inicial fue realizada por psiquiatras clínicos colaboradores con el hospital, especializados en poblaciones de adultos. Esto implica que los problemas habitualmente informados en niños/adolescentes, tales como problemas conductuales y emocionales, fuesen infrareportados. Además, el hecho que los participantes fuesen reclutados únicamente de un hospital psiquiátrico implica que los resultados no puedan ser generalizados a adolescentes en tratamiento hospitalario por uso de sustancias a nivel nacional. Por último, las relaciones observadas entre las variables y la duración temporal del tratamiento son solo correlacionales y carecen de la base necesaria para identificar la fuente o la dirección de la influencia.

\section{Conclusiones}

Los hallazgos destacan los perfiles complejos y múltiples de los adolescentes con problemas con el uso de sustancias en Brasil. Nuestros hallazgos señalan la necesidad de desarrollar enfoques para una colaboración entre los sistemas de salud mental, educación y servicios de justicia. Dada la duración temporal del tratamiento hospitalario y la ausencia de un modelo de atención continua después del alta tras el ingreso, este estudio ofrece evidencia que requiere ser considerada en el diseño de protocolos que ayuden a los adolescentes que intentan sortear sus perfiles problemáticos durante su recuperación.

\section{Conflicto de intereses}

Lo autores declaran la inexistencia de conflicto de intereses. Los autores asumen la autoría del contenido y la redacción de este documento.

\section{Referencias}

Bachman, J., O’Malley, P. M., Schulenberg, J. E., Johnston, L., Freedman-Doan, P. y Messersmith, E. E. (2008). The Education-Drug Use Connection: How Successes and Failures in School Relate to Adolescent Smoking, Drinking, Drug Use, and Delinquency. New York: Lawrence Erlbaum As- 
sociates. Recuperado de http:/ / www.psc.isr.umich.edu/ pubs/abs/4465.

Bastos, F. I. y Bertoli, N. (2014). Pesquisa Nacional sobre o uso de crack: quem são os usuários de crack e/ou similares do Brasil? Quantos são nas capitais brasileiras? Rio de Janeiro: Fundação Oswaldo Cruz. Recuperado de https://www.icict. fiocruz.br/sites/www.icict.fiocruz.br/files/Pesquisa\%20 Nacional\%20sobre\%20o\%20Uso\%20de\%20Crack.pdf.

Battjes, R. J., Gordon, M.S., O’Grady, K.E., Kinlock, T.W. y Carswell, M.A (2003). Factors that predict adolescent motivation for substance abuse treatment. Journal of Substance Abuse Treatment, 24, 221-32. doi:10.1016/ S0740-5472(03)00022-9.

Battjes, R. J., Gordon, M.S., O’Grady, K.E. y Kinlock T. W. (2004). Predicting retention of adolescents in substance abuse treatment. Addictive Behaviours, 29, 1021-1027. doi:10.1016/j.addbeh.2004.02.054.

Bertrand, K., Richer I., Brunelle, N., Beaudoi, I., Lemieux A. y Menard J. (2013). Substance Abuse Treatment for Adolescents: How are Family Factors Related to Substance Use Change? Journal of Psychoactive Drugs, 45, 28-38. doi:10.1080/02791072.2013.763560.

Broadfield D. (2017). Drug Misuse: Findings from the 2016/17 Crime Survey for England and Wales Statistical Bulletin 11/17. London: Home Office. Recuperado de https://www.gov.uk/government/uploads/system/ uploads/attachment_data/file/642738/drug-misuse2017-hosb1117.pdf.

Castellanos-Ryan, N., O'Leary冈Barrett, M., Sully, L. y Conrod, P. 2013). Sensitivity and specificity of a brief personality screening instrument in predicting future substance use, emotional, and behavioral problems: 18区 month predictive validity of the Substance Use Risk Profile Scale. Alcoholism: Clinical and Experimental Research, 37, E281-E290. doi:10.1111/j.1530-0277.2012.01931.x.

Carlini, E. L. A., Noto, A. R., Sanchez, Z. M., Carlini, C. M. A., Locatelli, D. P. y Abeid, L. R. (2010). VI Levantamento Nacional sobre o Consumo de Drogas Psicotrópicas entre Estudantes do Ensino Fundamental e Médio das Redes Pública e Privada de Ensino nas 27 Capitais Brasileiras. São Paulo: Centro Brasileiro de Informações sobre Drogas Psicotrópicas, SENAD - Secretaria Nacional de Políticas sobre Drogas.

Chung, T. y Maisto, S. A (2006). Relapse to alcohol and other drug use in treated adolescents: Review and reconsideration of relapse as a change point in clinical course. Clinical Psychology Review, 26, 149-161. doi:10.1016/j. cpr.2005.11.004.

Clark, D. y Winters, K. C. (2002). Measuring risks and outcomes in substance use disorders prevention research. Journal of Consulting and Clinical Psychology, 70, 12071223. doi:10.1037/0022-006X.70.6.1207.

Coatsworth, J. D., Santisteban, D. A., McBride, C. K. y Szapocznik J. (2001). Brief strategic family therapy versus community control: Engagement Retention, and an Ex- ploration of the Moderating Role of Adolescent Symptom Severity. Family Process, 40, 313-332. doi:10.1111/ j.1545-5300.2001.4030100313.x.

Cornelius, J. R., Maisto, S. A., Pollock, N. K., Martin, C. S., Salloum, I. M. y Lynch, K. G. (2003). Rapid relapse generally follows treatment for substance use disorders among adolescents. Addictive Behaviors, 28, 381-386. doi:10.1016/S0306-4603(01)00247-7.

Dennis, M. y Scott, C. K. (2007). Managing Addiction as a Chronic Condition. Addiction Science $\mathcal{E}$ Clinical Practice, 4, 45-55.

Kelly, J. F., Myers, M.G. y Brown, S.A. (2000). A Multivariate Process Model of Adolescent 12-Step Attendance and Substance Use Outcome Following Inpatient Treatment. Psychology of Addictive Behaviors, 14, 376-389.

Henry, K. L., Knight, K. E. y Thornberry, T. P. (2012). School Disengagement as a Predictor of Dropout, Delinquency, and Problem Substance Use during Adolescence and Early Adulthood. Journal of Youth Adolescent, 41, 156-166. doi:10.1007/s10964-011-9665-3.

Knudsen, H. K. (2009). Adolescent-only substance abuse treatment: availability and adoption of components of quality. Journal of Substance Abuse E Treatment, 36, 195204. doi:10.1016/j.jsat.2008.06.002.

Li, Y. y Lerner, R. M. (2011). Trajectories of school engagement during adolescence: Implications for grades, depression, delinquency, and substance use. Developmental Psychology, 47, 233-247. doi:10.1037/a0021307.

Lopes, G. M., Nobrega, B. A., Del Prette, G. y Scivoletto, S. (2013). Use of psychoactive substances by adolescents: current panorama. Revista Brasileira de Psiquiatria, 35, S51-S61. doi:10.1590/1516-4446-2013-S105.

Madruga, C., Laranjeira, R., Caetano, R., Pinsky, I., Zaleski, M. y Ferri, C. P. (2012). Use of licit and illicit substances among adolescents in Brazil - A national survey. Addictive Behaviours, 37, 1171-1175. doi:10.1016/j.addbeh.2012.05.008.

Malta, D. C., Mascarenhas, M. D. M., Porto, D. L., Duarte, E. A., Sardinha, L. M., Barreto, S. M. y Neto, O. L. M. (2011). Prevalência do consumo de álcool e drogas entre adolescentes: Análise dos dados da Pesquisa Nacional de Saúde Escolar. Revista Brasileira de Epidemiologia, 14, 136-146.

Mensinger, J. L., Diamond, G. S., Kaminer Y. y Wintersteen, M. B. (2006). Adolescent and Therapist Perception of Barriers to Outpatient Substance Abuse Treatment. American Journal on Addictions, 15, 16-25.

Miech, R. A., Johnston, L. D., O’Malley, P. M., Bachman, J. G. y Schulenberg, J. E. (2016). Monitoring the future national survey results on drug use, 1975-2015: Volume I, secondary school students. Ann Arbor, MI: Institute for Research for Social Research, The University of Michigan.

Myers, M. G., Brown, S. A. y Mott, M. A. (1995). Preadolescent conduct disorder behaviors predict relapse and 
progression of addiction for adolescent alcohol and drug abusers. Alcohol Clinical Experiment Research, 19, 1528 - 1536. doi:10.1111/j.1530-0277.1995.tb01019.x.

Paino, M., Aletraris, L. y Roman, P. M. (2015). Organizational Predictors and Use of Evidence-Based Practices in Adolescent Substance Abuse Treatment. Substance Abuse, 36, 462-469. doi:10.1080/08897077.2014.960959.

Plettinckx, E., Antoine, J., Blanckaert, P., De Ridder, K., Vander Laenen, F., Laudens, F.,... Gremeaux, L. (2014). Belgian National Report on drugs 2014, New Developments and Trends. Brussels: Belgian Monitoring Center for Drugs and Drugs Addiction. Recuperado de http:/ / www. emcdda.europa.eu/system/files/publications/1004/ BAR2014\% 20Final\%20EMCDDA\%20Version.pdf.

Roxburgh, A. y Burns, L. (2013). Drug-related hospital stays in Australia 1993-2013. Sydney: National Drug and Alcohol Research Centre. Recuperado de https://ndarc. med.unsw.edu.au/sites/default/files/ndarc/resources / NIDIP\% 20Bulletin \% 20-\% 20Drug-related \% 20hospital\%20stays\%20in\%20Australia\% 201993-2013.pdf.

Serrano, M. B., Al-Halabí, S., Burón, P., Garrido, M., Díaz-Mesa, E.M., Galván, G.,... Bobes J. (2018). Predictive factors of alcohol consumption in adolescents: data from 1-year follow-up prospective study. Adicciones. Avance de publicación on-line. doi:10.20882/adicciones.998.

Substance Abuse and Mental Health Services Administration. (2013). Results from the 2012 national survey on drug use and health: Summary of national findings. Rockville, MD: National Institutes of Health.

Svensson, R. (2000). Risk factors for different dimensions of adolescent drug use. Journal of Child $\mathcal{E}$ Adolescent Substance Abuse, 9, 67-90. doi:10.1300/J029v09n03_05.

Sussman, S., Skara, S. y Ames, S. L. (2009). Substance abuse among adolescentes. Substance Use EF Misuse, 43, 18021828. doi:10.1080/10826080802297302.

Vasters, G. P. y Pillon S. (2011). O uso de drogas por adolescentes e suas percepções sobre adesão e abandono de tratamento especializado. Revista Latino-Americana de Enfermagem, 19. doi:10.1590/S010411692011000200013.

NIDA. (2014). Principles of Adolescent Substance Use Disorder Treatment: A Research-Based Guide. Rockville, MD: National Institutes of Health. Recuperado de https://d14rmgtrwzf5a.cloudfront.net/sites/default/files/podata_1_17_14.pdf.

Winters, K. C., Botzet, A. M., Stinchfield, R., Gonzales-Castaneda, R., Finch, A. J., Piehler, T. F.,... Hemze, A. (2018). Adolescent Substance Abuse Treatment: A Review of Evidence-Based Research. In C. G. Leukefeld \& T. P. Gullotta (Eds.). Adolescent Substance Abuse. Evidence-Based Approaches to Prevention and Treatment. (pp. 141171). New York, NY: Springer Science

Winters, K. C., Stinchfield, R. D., Opland, E., Weller, C. y Latimer, W. W. (2000). The effectiveness of the Minnesota Model approach in the treatment of adolescent drug abusers. Addiction, 95, 601-612. doi:10.1046/j.13600443.2000.95460111.x.

Winters, K. C., Tanner-Smith, E. E., Bresani, E. y Meyers K. (2014). Current advances in the treatment of adolescent drug use. Adolescent Health, Medicine Therapeutics, 5, 199210. doi:10.2147\%2FAHMT.S48053. 